\title{
Educación a distancia y educación virtual: una diferencia necesaria desde la perspectiva pedagógica y la formación del ser humano
}

\author{
Nubia Constanza Arias Arias ${ }^{1}$ \\ Karolina González Guerrero ${ }^{2}$ \\ José Eduardo Padilla Beltrán ${ }^{3}$
}

\begin{abstract}
Resumen
La educación a distancia y la educación virtual en la actualidad se constituyen en escenarios educativos que marcan una diferencia desde la perspectiva pedagógica y de formación del ser humano. Según lo expuesto en el Decreto 1295 del 20 de abril de 2010, los programas de educación a distancia son aquellos "cuya metodología educativa se caracteriza por utilizar estrategias de enseñanza - aprendizaje que permiten superar las limitaciones de espacio y tiempo entre los actores del proceso educativo (Ministerio de Educación Nacional, 2010). De igual manera el mismo decreto establece que "Los programas virtuales, adicionalmente, exigen el uso de las redes telemáticas como entorno principal, en el cual se lleven a cabo todas o al menos el ochenta por ciento $(80 \%)$ de las actividades académicas".
\end{abstract}

\footnotetext{
1 Licenciada en Psicología y Pedagogía, Universidad Pedagógica Nacional; Especialista en Educación Sexual, Fundación Universitaria Los Libertadores; Doctora en Educación Universidad de Oviedo (España). Coordinadora de Autoevaluación y Acreditación Institucional, Coordinadora del Programa de Maestría en Educación. Grupo de Investigación Pedagogía y Didáctica de la Educación superior, PYDES. Docente de planta de la Universidad Militar Nueva Granada.

2 Licenciada en electrónica, Universidad Pedagógica Nacional; Magister en Educación, Pontificia Universidad Javeriana; Candidato a Doctor en Educación y Multimedia, Universidad de Aveiro (Portugal). Editora Revista Educación y Desarrollo Social. Grupo de Investigación Pedagogía y Didáctica de la Educación superior (PYDES). Docente de planta de la Universidad Militar Nueva Granada.

3 Licenciado en Matemáticas, Universidad Pedagógica Nacional; Especialista en Orientación Educativa, Universidad Manuela Beltrán; Magíster en Administración y Supervisión Educativa, Universidad Externado de Colombia; Magíster en Educación con énfasis en Evaluación Educativa, Universidad Santo Tomás; Philosophical Doctor, Newport University, USA. Director del Departamento de Educación, Universidad Militar Nueva Granada. Presidente de Ascofade, Capítulo Centro. Líder del Grupo de Investigación en Pedagogía y Didáctica de la Educación Superior, PYDES.
} 
De acuerdo con las definiciones expuestas, es necesario hacer una profunda reflexión desde el punto de vista pedagógico, que permita clarificar la influencia de las metodologías o ambientes virtuales de aprendizaje en la formación integral del ser humano, más allá de lo cognitivo; el artículo presentado pretende desarrollar una reflexión pedagógica sobre la formación del ser humano en la metodología a distancia y los entornos virtuales de aprendizaje.

Se espera que las ideas expuestas propicien la discusión sobre el verdadero papel del maestro o tutor en los entornos descritos y las competencias básicas y profesionales que debe poseer como persona para atender a la formación de personas y profesionales en estos tipos de educación. La comprensión del sentido, significado, funciones y prácticas de la educación a distancia y la educación virtual, requiere investigación educativa y pedagógica que logre avances

significativos en la formación integral del ser humano en contextos distintos a la presencialidad.

Por lo mencionado, proponemos como objeto de estudio desde una perspectiva pedagógica crítica, las problemáticas presentadas en la educación a distancia y virtual evidenciando los logros, contradicciones y conflictos en torno a estas metodologías, e invitando a la reflexión, el diálogo y el razonamiento dialéctico de docentes investigadores y grupos de estudio interesados en darle un carácter formativo, formador y pedagógico a las metodologías que para algunos se presentan como el futuro de la educación.

Palabras clave: educación a distancia, educación virtual, profesión docente, investigación pedagógica, formación del ser humano.

\title{
Distance and virtual education: a necessary difference from the teaching and the training perspectives
}

\begin{abstract}
Distance and Virtual Education are currently the educational settings that make a difference from a pedagogical perspective and training of human
\end{abstract}


beings. As set out in Decree 1295 of April 20, 2010, the distance education programs are those "whose educational methodology is characterized by using teaching strategies - learning to overcome the limitations of space and time between the actors of the educational process. Similarly, the same Decree states that "virtual programs, in addition, require the use of such networks as the primary environment in which to carry out all or at least eighty percent $(80 \%)$ of academic activities".

According to the above definitions is necessary to make a deep reflection from the pedagogical point of view, that clarifies the influence of the methodologies or virtual learning environments in the formation of the human being beyond the cognition; the paper presented is to develop pedagogical reflection on the training of human beings in the methodology for distance and virtual learning environments.

It is hoped that the ideas support to the discussion about the real role of teacher or tutor in the described environments and the basic and professional required competences he must possess as a person in order to attend to people and professionals in these types of education. Comprehension about sense, significance, functions and practices of distance education and virtual education requires educational and pedagogical research becomes a significant progress in the integral formation of human beings in contexts other than being present.

As mentioned above, we propose as an object of study from a critical and pedagogical perspective, the issues presented in the Distance and Virtual Education, highlighting the achievements, contradictions and conflicts over these methodologies, and inviting the teacher researchers and study groups to reflection, dialogue and dialectical reasoning in order to insert an educational and pedagogical character to teaching methodologies being introduced as the future of education.

Key words: distance education, virtual education, teaching profession, educational research, training of human beings.

\section{Introducción}

El presente artículo sintetiza aportes que la investigación pedagógica hace para la configuración de las finalidades que deben tener tanto la educación a distancia 
como la educación virtual en el contexto de la formación integral de los seres humanos, evidenciando los requerimientos que el docente y el estudiante deben tener para desarrollar un proceso de enseñanza-aprendizaje mediante esta metodología y en este ambiente de aprendizaje.

Desde la perspectiva expuesta, la investigación pedagógica para la educación a distancia y la educación virtual se constituye en una oportunidad de transformación y resignificación permanente del trabajo pedagógico, teniendo en cuenta los avances investigativos para concebir este tipo de educación como un contexto de comprensión, investigación, negociación, diálogo y transformación de situaciones conflictivas o de crisis que contribuyan a la formación integral de los seres humanos.

La disertación expuesta se constituye en una reflexión para aquellos que se desenvuelven como docentes o estudiantes en los contextos de la educación a distancia o virtual y que a su vez creen en la formación integral del ser humano más allá de la presencialidad.

\section{Consideraciones preliminares}

Las investigaciones y los expertos en el uso de los medios y tecnologías de la información y la comunicación aplicadas en la educación, MTIC, coinciden en afirmar que en el contexto actual la virtualidad se constituye en un escenario básico para comprender los procesos de enseñanza-aprendizaje, las mediaciones, el papel del docente como mediador, el papel del estudiante, entre otros aspectos del ámbito educativo. Es por ello, fundamental considerar el sentido y significado de la virtualidad y sus finalidades tanto para la educación presencial como para la educación a distancia.

La virtualidad, según Pérez (2010a), tiene como finalidades básicas las siguientes:

a) Suministro de información: Cuando se hace referencia al suministro de información se explicita que la virtualidad en el proceso de enseñanzaaprendizaje puede llevar al estudiante una información básica, actualizada, pertinente sobre los avances científicos y tecnológicos de cada una de las áreas del conocimiento. No obstante, la información como tal no es suficiente, ya que se requiere la medición del docente para que esta información se transforme en conocimiento que le permita al individuo actuar con criterio en situaciones cotidianas y profesionales.

b) Comunicación: La comunicación en el marco de la virtualidad es una de las finalidades esenciales del proceso educativo, por lo cual el espacio virtual tiene que permitir tanto al docente como a los estudiantes generar procesos de comunicación asertiva, que les den la posibilidad de construir conocimiento, intercambiar experiencias, formar valores y actitudes. Esto 
no sólo se logra a través del espacio virtual sino que del mismo modo los encuentros personales en un espacio físico son necesarios para esta formación que a nivel curricular va más allá de la transferencia de información y alcanza la integralidad del ser.

c) Simulación o entrenamiento: La virtualidad como espacio de construcción de conocimiento, actitudes, aptitudes y valores proporciona al individuo entornos de aprendizaje experiencial. Esto implica que la virtualidad suministra desde una perspectiva pedagógica escenarios donde el estudiante de cualquier área experimente permanentemente el entorno de trabajo profesional, desarrollando habilidades, actitudes, valores y conocimientos, con ejercicios de simulación o entrenamiento que lo sometan a las condiciones de desempeño propios del trabajo que desarrollará en su vida profesional. Lo descrito es complementario con la práctica en la realidad, propia de los campos ocupacionales donde se desempeñará en el futuro.

Requisitos para el desarrollo de la virtualidad en procesos educativos y pedagógicos

Teniendo claridad en las finalidades de la virtualidad en la educación que enmarcan el sentido y significado del uso de los medios y tecnologías de la información y la comunicación (MTIC), es primordial comprender que la virtualidad sólo es una realidad si cumple a cabalidad con los siguientes requisitos:

Institucionales: La institución educativa dispondrá de la infraestructura física y tecnológica y de los recursos humanos, físicos y financieros para garantizar el desarrollo de los programas académicos que hagan uso de la virtualidad como espacio de formación de los estudiantes. Por ello es imprescindible que cuente con:

- Espacios físicos suficientes y adecuados para el número de participantes en sesiones de trabajo presencial, sea en las instalaciones de la propia institución o en convenio con otras instituciones que sean de fácil acceso a los estudiantes en proceso de formación.

- Equipos tecnológicos e informáticos, redes y software que permitan el suministro de información actualizada, la comunicación y la simulación o entrenamiento para los actores del proceso de enseñanza-aprendizaje de manera eficiente, eficaz y oportuna.

- Mantenimiento permanente tanto de los espacios físicos como de la infraestructura tecnológica e informática, que permita la solución inmediata de las situaciones problemáticas que se puedan presentar en el desarrollo del proceso de enseñanza aprendizaje. 
- Recursos humanos suficientes y calificados que respondan con oportunidad a las necesidades de los beneficiarios de los programas de formación, dando retroalimentación permanente a las personas durante su proceso de formación.

Docentes: El educador, según la Resolución 5443 del Ministerio de Educación (2010), es un profesional con formación pedagógica, que tiene en cuenta los contextos y las condiciones personales del educando para el desarrollo de sus competencias. Por supuesto, las personas que acompañan, orientan, dirigen y forman a los estudiantes son personas con las condiciones y calidades requeridas para el desempeño profesional de un educador en el contexto actual. Es por ello que los docentes, mediadores del aprendizaje en contextos de virtualidad, como mínimo cumplirán con los siguientes requisitos:

- Ser idóneo en la disciplina o área de conocimiento que enseñan.

- Poseer un nivel avanzado de formación pedagógica.

- Poseer un nivel avanzado de formación en tecnologías de la información y la comunicación.

- Tener capacidad y disposición de trabajo colaborativo y en equipo.

Las condiciones descritas serán certificadas debidamente, al tiempo que serán evaluadas constantemente con miras a la cualificación permanente y a la superación de las falencias detectadas en el proceso.

Estudiantes: Las personas que vivencia la virtualidad como un espacio de formación requieren preparación y ambientación adecuadas a este entorno. Comúnmente han sido formadas en la educación tradicional que tiene fundamentos epistemológicos y pedagógicos donde priman, por lo general, la transmisión de la información, la autoridad del docente y en consecuencia, la poca autonomía del estudiante y el escaso empleo de las tecnologías, entre otros aspectos. Por lo descrito, el estudiante que asume un proceso de formación en entornos virtuales, tendrá que disponer de:

a) Motivación y conocimiento de las condiciones del proceso de enseñanza-aprendizaje.

Esto implica que reciba formación en el desarrollo del aprendizaje autónomo y que comprenda su rol como estudiante y el del docente, trazándose a la vez una proyección de vida que le permita percibirse en su cotidianidad en el entorno de la virtualidad, lo que se traduce en aprender a comunicarse en este espacio, realizar trabajos de simulación y entrenamiento en el mismo, obtener información 
y tener criterio para seleccionar de ella lo más adecuado a sus requerimientos contextuales, entre otros aspectos.

b) Facilidad de acceso a las mediaciones tecnológicas de la información y la comunicación.

Factor fundamental que marca el proceso de enseñanza aprendizaje en contextos de virtualidad es el acceso que la persona tenga a las tecnologías. Por ello la institución debe proveer de espacio para que la persona en su contexto pueda llegar con facilidad a las MTIC.

\section{c) Capacitación en el uso de las MTIC.}

En el contexto actual un gran número de personas convive cotidianamente con las tecnologías de la comunicación y la información. Sin embargo, esta convivencia no implica que la persona tenga un conocimiento preciso y profundo de las oportunidades que ofrecen estos espacios virtuales para el proceso enseñanzaaprendizaje. Padilla afirma: "un talento humano capacitado podrá dar mejores resultados y un mayor sentido a la incorporación de las diferentes tecnologías en el proceso de formación" (2008, p.106). Según lo descrito, es fundamental formar continuamente a estudiantes y profesores en el uso de las MTIC y en los avances tecnológicos que enriquecen la virtualidad, para hacer más efectivo y productivo el proceso de enseñanza-aprendizaje para el futuro desempeño profesional de los estudiantes y la aplicación de las MTIC en la cotidianidad y en la proyección de su vida.

\section{Condiciones para el desarrollo de la virtualidad en programas de educación superior presenciales y a distancia}

En consideración a los requisitos presentados es pertinente aclarar que la virtualidad al ser empleada tanto en la educación presencial como en la educación a distancia, tendría que ser considerada tanto en el diseño curricular como en la planificación de las actividades cotidianas de enseñanza-aprendizaje.

Particularmente y en razón del Decreto 1295 del 20 de Abril de 2010 en su Capítulo VI, es necesario presentar, si se ofrecen programas a distancia o virtuales, una serie de requisitos adicionales a las condiciones mínimas de los programas presenciales, que contribuyen a la calidad.

En cuanto al manejo de la metodología y el desarrollo de competencias, se puede observar que las instituciones de educación superior deben garantizar "la forma como desarrollarán las actividades de formación académica, la utilización efectiva de mediaciones pedagógicas y didácticas, y el uso de formas de interacción apropiadas que apoyen y fomenten el desarrollo de competencias para el 
aprendizaje autónomo“ (Artículo 18), lo cual reclama la propuesta de seminarios inductivos al inicio de las carreras profesionales.

En relación con espacios físicos para el desarrollo de las prácticas, clínicas o talleres y la relación con la empresa o industria se pretende que las IES “deben indicar la infraestructura, medios educativos y personal docente de los lugares donde se desarrollen dichas prácticas" (Artículo 19). Es decir, hay un compromiso alto de la IES en garatizar los standares de oportunidad para la adquisición de competencias tanto laborales como profesionales del educando. Igualmente, la IES debe "suministrar información pertinente a la comunidad sobre los requerimientos tecnológicos y de conectividad necesarios para cursar el programa".

De acuerdo con lo establecido en el decreto antes mencionado, en los programas ofrecidos a distancia, los documentos maestros para la presentación del registro calificado y la acreditación de alta calidad deben referenciar específicamente:

- La forma como desarrollarán las actividades de formación académica.

- La utilización efectiva de mediaciones pedagógicas y didácticas.

- El uso de formas de interacción apropiadas que apoyen y fomenten el desarrollo de competencias para el aprendizaje autónomo.

- La realización de prácticas, clínicas o talleres o la presencia de los estudiantes en centros de tutoría.

Teniendo en cuenta lo descrito para el desarrollo de las actividades de formación académica, los programas de Educación a Distancia deberán contar con las siguientes condiciones:

\section{En el ámbito institucional - administrativo}

- Infraestructura, medios educativos y personal docente en los lugares donde se desarrollarán las actividades de formación académica y prácticas entre otros, sea en la sede principal o en centros regionales (convenios nacionales e internacionales).

- Efectividad en recursos empleados en las mediciones pedagógicas y didácticas y el mejor aprovechamiento de los recursos con relación a la distribución de horarios y el tiempo destinado para la atención a estudiantes.

- Clima organizacional que genere entre los miembros de la comunidad educativa y académica la motivación y el avance en el desarrollo de las funciones de docencia, investigación, extensión y proyección social, propiciando así, el trabajo en equipo. 
- Evaluación de estudiantes sobre el aprendizaje autónomo y el uso de las MTIC, con cuyos resultados se puedan plantear acciones de mejoramiento a corto, mediano y largo plazo.

- Definición de criterios y pautas claras de gestión en trámites académicoadministrativos y de calendarios académicos organizados y precisos que permitan desarrollar en los estudiantes, valores como la responsabilidad, el compromiso y la honestidad, entre otros contemplados en los principios y valores de la comunidad educativa.

- Apoyo para la autoevaluación y plan de mejoramiento de programas para la obtención del registro calificado, renovación del mismo o acreditación de alta calidad, con las condiciones contempladas en el Decreto 1295 de abril de 2010 y en la Resolución 5443 del 30 de junio de 2010.

\section{En los procesos de enseñanza y aprendizaje}

El desarrollo de los procesos de enseñanza-aprendizaje conducentes a la formación de seres humanos integrales requiere en el contexto actual la conciencia de la intencionalidad, sentido y significado del proceso educativo mediado por los medios y tecnologías de la información y la comunicación. Esto exige que, así como en la educación presencial formalizada, la organización de procesos educativos a distancia o virtuales se sometan a un análisis desde el punto de vista pedagógico que involucre los siguientes aspectos:

La planificación de las actividades académicas: Implica realizar una labor de prospectiva de la formación y los requerimientos de la misma, de acuerdo con el contexto local, regional, nacional e internacional. Por ello, la preparación del diseño de proyectos curriculares requiere, según Pérez (2010b), definir las fuentes o fundamentos del currículo entre los que se destacan: la epistemológica, la investigativa, la axiológica, la antropológica, la sociológica, la psicológica, la pedagógica, la didáctica y la etnográfica

Estas fuentes permiten visualizar el ideal del ser humano que se pretende formar, enmarcado en un contexto institucional. A partir de estos fundamentos se establecen los componentes del currículo, que desarrollan en la práctica el ideal del ser humano tanto social como cultural e institucional.

Con respecto a los componentes del currículo en la educación a distancia y virtual, se pueden considerar finalidades, objetivos del programa, perfiles de ingreso, proceso y egreso de las personas a quienes se pretende formar y las competencias.

De igual forma se relacionan los contenidos, la metodología, los recursos y medios audiovisuales, la formación investigativa, los espacios tanto físicos como 
virtuales, la temporalización y la evaluación teniendo en cuenta los procesos y los resultados.

Tabla 1. Similitud en porcentajes de estrategias didácticas entre las actividades presenciales y a distancia

\begin{tabular}{|l|l|l|}
\hline ESTRATEGIAS DIDÁCTICAS & PRESENCIAL & DISTANCIA \\
\hline Clase magistral & $8 \%$ & $8 \%$ \\
\hline Conferencia experto & $2 \%$ & $2 \%$ \\
\hline Foro, debate, panel & $12 \%$ & $12 \%$ \\
\hline Lecturas guiadas & $12 \%$ & $12 \%$ \\
\hline Seminario & $16 \%$ & $16 \%$ \\
\hline Trabajo en grupo & $10 \%$ & $10 \%$ \\
\hline Estudio de caso & $12 \%$ & $12 \%$ \\
\hline Resolución de problemas & $10 \%$ & $10 \%$ \\
\hline Programas de intervención & $5 \%$ & $5 \%$ \\
\hline Proyectos de Investigación & $8 \%$ & $8 \%$ \\
\hline Otra: Tutoría grupal, etc. & $5 \%$ & $5 \%$ \\
\hline PORCENTAJE TOTAL & $100 \%$ & $100 \%$ \\
\hline
\end{tabular}

Se muestra entonces cómo a través del sistema de créditos, la virtualidad es un escenario primordial en la educación a distancia, ya que se presenta como una mediación que crea un espacio de interacción permanente, comunicación, suministro de información y entretenimiento. Según estas posibilidades, la ponderación del número de estrategias utilizadas tanto en lo presencial como en lo virtual son iguales, como se ve en la tabla 1 .

El papel de los actores en los procesos de enseñanza-aprendizaje: El trabajo interdisciplinario del grupo de docentes es un aspecto decisivo en la puesta en práctica de la educación a distancia o virtual, por lo cual, los módulos, guías o lineamientos para el aprendizaje deben ser fruto del trabajo de un grupo interdisciplinario conformado por: el docente, expertos del área de conocimiento del programa de formación ofrecido, un especialista en pedagogía, didáctica, currículo y evaluación y un especialista en uso de las mediaciones tecnológicas de la información y la comunicación. Los módulos y el material de apoyo didáctico deberán ser renovados al menos cada semestre, con el apoyo de los docentes y los estudiantes para mantenerlos actualizados y con los últimos avances del área del saber o disciplina.

\section{Actividades de formación con el uso de TIC}

Para apreciar el desarrollo de las actividades de formación con el uso de las mediaciones tecnológicas de la comunicación y la información, a continuación se presentan las funciones educativas (Marqués, 2010): 
Tabla 1. Similitud en porcentajes de estrategias didácticas entre las actividades presenciales y a distancia

\begin{tabular}{|c|c|}
\hline \multicolumn{2}{|c|}{ FUNCIONES EDUCATIVAS DE LAS MTIC y LOS "MASS MEDIA" } \\
\hline FUNCIONES & INSTRUMENTOS \\
\hline $\begin{array}{l}\text { Medio de expresión y creación multimedia, } \\
\text { para escribir, dibujar, realizar presentaciones } \\
\text { multimedia y elaborar páginas web. }\end{array}$ & $\begin{array}{l}\text { - Procesadores de textos, editores de imagen } \\
\text { y vídeo, editores de sonido, programas de } \\
\text { presentaciones, editores de páginas web. } \\
\text { - Lenguajes de autor para crear materiales } \\
\text { didácticos interactivos. } \\
\text { - Cámara fotográfica, vídeo. } \\
\text { - Sistemas de edición videográfica, digital y } \\
\text { analógica. }\end{array}$ \\
\hline $\begin{array}{l}\text { canal de comunicación, que facilita la } \\
\text { comunicación interpersonal, el intercambio de } \\
\text { ideas y materiales y el trabajo colaborativo. }\end{array}$ & $\begin{array}{l}\text { - Correo electrónico, Chat, videoconferencias, } \\
\text { listas de discusión, foros... }\end{array}$ \\
\hline $\begin{array}{l}\text { Instrumento de productividad para el proceso de } \\
\text { la información: crear bases de datos, preparar } \\
\text { informes, realizar cálculos... }\end{array}$ & $\begin{array}{l}\text { - Hojas de cálculo, gestores de bases de datos. } \\
\text { - Lenguajes de programación. } \\
\text { - Programas para el tratamiento digital de la } \\
\text { imagen y el sonido. }\end{array}$ \\
\hline $\begin{array}{l}\text { Fuente abierta de información y de recursos } \\
\text { (lúdicos, formativos, profesionales). En el caso } \\
\text { de Internet, hay "buscadores" especializados } \\
\text { para ayudarnos a localizar la información que } \\
\text { buscamos. }\end{array}$ & $\begin{array}{l}\text { - CD-ROM, vídeos DVD, páginas Web de interés } \\
\text { educativo en Internet... } \\
\text { - Prensa, radio, televisión. }\end{array}$ \\
\hline $\begin{array}{l}\text { Instrumento cognitivo que puede apoyar } \\
\text { determinados procesos mentales de los } \\
\text { estudiantes asumiendo aspectos de una tarea: } \\
\text { memoria que le proporciona datos para comparar } \\
\text { diversos puntos de vista, simulador donde probar } \\
\text { hipótesis, entorno social para colaborar con } \\
\text { otros, proveedor de herramientas que facilitan la } \\
\text { articulación y representación de conocimientos. }\end{array}$ & $\begin{array}{l}\text { - Todos los instrumentos anteriores, considerados } \\
\text { desde esta perspectiva como instrumentos de } \\
\text { apoyo a los procesos cognitivos del estudiante. } \\
\text { - Generador de mapas conceptuales. }\end{array}$ \\
\hline $\begin{array}{l}\text { Instrumento para la gestión administrativa y } \\
\text { tutorial }\end{array}$ & $\begin{array}{l}\text { - Programas específicos para la gestión de } \\
\text { centros y } \\
\text { seguimiento de tutorías. } \\
\text { - Web del centro con formularios para facilitar la } \\
\text { realización de trámites online. }\end{array}$ \\
\hline $\begin{array}{l}\text { Herramienta para la orientación, el diagnóstico y } \\
\text { la rehabilitación de estudiantes. }\end{array}$ & $\begin{array}{l}\text { - Programas específicos de orientación, diag- } \\
\text { nóstico y } \\
\text { rehabilitación. } \\
\text { - Webs específicos de información para la } \\
\text { orientación } \\
\text { escolar y profesional. }\end{array}$ \\
\hline
\end{tabular}




\begin{tabular}{|l|l|}
\hline $\begin{array}{l}\text { Medio didáctico y para la evaluación: informa, } \\
\text { ejercita habilidades, hace preguntas, guía el } \\
\text { aprendizaje, motiva, evalúa... }\end{array}$ & $\begin{array}{l}\text { - Materiales didácticos multimedia (soporte disco } \\
\text { o en } \\
\text { Internet). } \\
\text { - Simulaciones. } \\
\text { - Programas educativos de radio, vídeo y tele- } \\
\text { visión. } \\
\text { - Materiales didácticos en la prensa. }\end{array}$ \\
\hline $\begin{array}{l}\text { Instrumento para la evaluación, que propor- } \\
\text { ciona: corrección rápida y feedback inmediato, } \\
\text { reducción de tiempos y costos, posibilidad de } \\
\text { seguir el "rastro" del estudiante, uso en cualquier } \\
\text { ordenador (si es online). }\end{array}$ & $\begin{array}{l}\text { - Programas y páginas web interactivas para } \\
\text { evaluar conocimientos y habilidades. }\end{array}$ \\
\hline $\begin{array}{l}\text { Soporte de nuevos escenarios formativos. } \\
\text { Medio lúdico y para el desarrollo cognitivo. }\end{array}$ & $\begin{array}{l}\text { - Entornos virtuales de enseñanza. } \\
\text { - Prensa, radio, televisión... }\end{array}$ \\
\hline
\end{tabular}

Fuente: Marqués, P. (2000), “Impacto de las TIC en el mundo educativo" (en línea), disponible en: http:// peremarques. pangea.org/siyedu.htm, recuperado: 18 de julio de 2010)

La relación orientada anteriormente, permite diferenciar entre funciones e instrumentos y orienta al equipo docente para tomar alternativas que optimicen las estrategias de aprendizaje. Igualmente, se clasifica cada una de las funciones con el propósito de hacer conciencia del uso del instrumento y el impacto de las mediaciones.

La evaluación del proceso de educativo: Se recomienda la evaluación permanente de los componentes del proceso educativo: los recursos y plataformas virtuales, docentes, estudiantes, procesos de comunicación, formación de comunidad académica, actualización y pertinencia de la información proporcionada en las diferentes disciplinas y área de conocimiento para establecer planes de mejoramiento que aseguren la calidad en el servicio educativo.

Para la evaluación de las MTIC empleadas en el ámbito educativo, se recomienda analizar los puntos propuestos en el decálogo presentado por Area (2008) sobre su uso didáctico en el aula, quien recomienda:

1. Lo relevante debe ser siempre lo educativo, no lo tecnológico.

2. Las MTIC no tienen efectos mágicos sobre el aprendizaje ni generan automáticamente innovación educativa (ni se es mejor o peor profesor, ni los alumnos aumentan motivación, interés o rendimiento). 
3. El método o estrategia didáctica junto con las actividades planificadas, son los que promueven un tipo u otro de aprendizaje (recepción, descubrimiento).

4. Los estudiantes deben hacer cosas con la tecnología.

5. Las MTIC deben usarse como recursos de apoyo para el aprendizaje académico de las distintas materias curriculares, y para la adquisición y desarrollo de competencias específicas en MTIC.

6. Las MTIC pueden usarse tanto para la búsqueda, consulta y elaboración de información como para relacionarse y comunicarse con otras personas (tareas intelectuales y sociales).

7. Las MTIC se deben utilizar tanto para el trabajo individual como para el desarrollo de procesos de aprendizaje colaborativo entre grupos de alumnos (presencial y virtualmente).

8. Cuando se planifica una lección, proyecto o actividad con MTIC, debe explicitarse tanto el objetivo y contenido del aprendizaje curricular como el tipo de competencia o habilidad tecnológica que promueve.

9. Evitar la improvisación en el aula de informática. Planificar tareas, grupos, proceso de trabajo, tiempos.

10. El uso de las MTIC no debe planificarse como una acción paralela al proceso de enseñanza habitual sino que se debe integrar.

En las instituciones de educación superior que ofrecen programas mediante la modalidad a distancia o virtual, los docentes hacen las veces de autor, tutor o formador. En este sentido, afirma Padilla (2008) que el rol del docente es fundamental ya que no solo debe ser idóneo en la disciplina o saber que orienta sino, además, en el manejo con nivel de experticia de las mediaciones tecnológicas de comunicación e información.

\section{Conclusión}

Para desarrollar la virtualidad en los programas de educación superior presenciales y a distancia es necesario:

Que cada uno de los miembros participantes en el proceso educativo tenga formación y actualización permanente en los avances de las tecnologías de la información y la comunicación, para que éstas puedan emplearse en el 
proceso pedagógico para suministrar información, comunicación, simulación o entrenamiento en el área profesional propia de cada disciplina.

Que el tutor, formador o educador no pierda el horizonte de su misión con relación a la formación de los seres humanos, por lo cual más allá de suministrar información, se ocupa de construir horizontes de vida, prospectivas y conocimientos para la vida misma.

Que el estudiante desarrolle procesos de autogestión, autorregulación, autonomía, pensamiento crítico y creativo; por ello, antes que brindar información, la educación presencial o a distancia tiene que encaminarse al desarrollo de los mencionados procesos.

Que la institución de educación disponga de espacios presenciales y virtuales dotados y mantenidos adecuadamente, para contribuir a la formación y desarrollo humano de los miembros de la comunidad educativa.

Que en la planificación, desarrollo y evaluación de los programas de educación superior presenciales y a distancia, se incluya la virtualidad como elemento inherente, que permita considerar a cada individuo en relación con los demás actores del proceso educativo, propiciando espacios de intercambio de información, comunicación, y simulación de experiencias propias de la vida cotidiana.

Téngase en cuenta que en la formación y el desarrollo humano lo relevante debe ser siempre lo educativo y lo pedagógico, no lo tecnológico, bien sea educación presencial, a distancia o virtual.

\section{Referencias bibliográficas}

Andrade, L. (2007). “Ambientes de aprendizaje para la educación en tecnología”, Departamento de Tecnología, Universidad Pedagógica Nacional, Bogotá.

Area, M. (2008). "La innovación pedagógica con TIC y el desarrollo de las competencias informaciones y digitales”. En Investigación en la Escuela. № 64 .

Asociación Colombiana de Universidades - Ascun, Instituto Colombiano para el Fomento de la Educación Superior - Icfes. Seminario Construyendo la universidad virtual. Bogotá, 23-34 de septiembre de 2002.

Facundo, Ángel. Educación virtual en América Latina y el Caribe: características y tendencias. Bogotá: Unesco/IESALC, febrero 2002. 
Galliani, L. (2002). L'università aperta e virtuali, pensa multimedia, Lecce.

González, Luis José y otros. La educación superior a distancia en Colombia. Visión histórica y lineamientos para su gestión. Bogotá: Icfes, 2000, p. 25.

Icfes, Elementos de política para la educación superior colombiana. Memorias de gestión y prospectiva. Bogotá: Icfes, 2002.

Internacional Council For Open And Distance Education - Icde y Consorcio de Universidades del Perú. El impacto social de las nuevas tecnologías en la educación a distancia en América Latina y el Caribe. Quinta reunión regional de educación a distancia. Lima, 14-16 de noviembre de 2002.

Maldonado, Luis F. Nuevas metodologías aplicadas a la educación. Estado del arte de la investigación 1990-1999. En: Colciencias. Estados del arte de la investigación en educación y pedagogía en Colombia. Bogotá: Icfes, Colciencias, Sociedad Colombiana de Pedagogía, Tomo II. 2001.

Marqués, P. (2000). “Impacto de las TIC en el mundo educativo” (en línea), disponible en: http://peremarques.pangea.org/siyedu.htm, recuperado: 18 de julio de 2010.

Ministerio de Educación Nacional. Decreto 1295 de 2010 "Por el cual se reglamenta el registro calificado de que trata la Ley 1188 de 2008 y la oferta y desarrollo de programas académicos de educación superior".

Resolución 5443 de 2010 "Por la cual se definen las características especificas de calidad de los programas de formación profesional en educación”.

Padilla, J. E. (2008). Globalización y Educación Superior: Un reto en la formación del docente universitario, Bogotá: Editorial Bonaventuriano.

Pérez, R. (2000). Redes, multimedia y diseños virtuales, Oviedo: Universidad de Oviedo.

(2010a). "La virtualización de la educación superior: límites y posibilidades en el contexto de la responsabilidad social”, II congreso internacional de investigación educativa y pedagógica. Bogotá: UMNG.

(2010b). Diseño de proyectos curriculares. Oviedo: Universidad de Oviedo. 